\section{El Internet y su incorporación al sector de la salud}

\author{
Carlos Linger, ${ }^{1}$ Hugo Spinelli ${ }^{2} y$ \\ Celia Iriart $^{3}$
}

1 Representante de la OPS en La Paz, Bolivia. Dirección postal: Edificio "Foncomin", Av. 20 de Octubre \#2038, $3^{\text {er }}$ piso, La Paz, Bolivia.

2 Consultor, Organización Panamericana de la Salud, Buenos Aires, Argentina.

3 Consultora, Organización Panamericana de la Salud, Buenos Aires, Argentina.
La mayor parte de los países de América Latina, que se encuentran actualmente en proceso de hacer reformas estatales, necesitan recapacitar al personal de la salud a costos relativamente bajos y en plazos cortos sin sacrificar la calidad de la capacitación. Desde que se adoptó en los años ochenta en la Región de las Américas la política denominada Educación Permanente en Salud (EPS) se viene señalando la necesidad de exponer a todo el personal de la salud a metodologías educativas que tengan un impacto en el proceso de trabajo. No obstante, esta propuesta es difícil de llevar a la práctica de forma generalizada debido a los ajustes estructurales con recortes del gasto público que se están llevando a cabo en la mayor parte de los países.

En los últimos años, el campo de la comunicación y el acceso a información se han visto radicalmente transformados por redes como el Internet. El acelerado desarrollo de programas que facilitan su uso ha despertado el interés de millones de usuarios.

En el campo de la salud, numerosos profesionales y técnicos utilizan el Internet como medio de actualización y de comunicación e información sobre temas de su interés. De hecho, han surgido redes sobre temas de salud específicos que ayudan a concentrar la información, pero que al mismo tiempo fragmentan su disponibilidad. Estas redes pueden llevar a la creación de "feudos", ya que el rápido crecimiento general de la red dificulta la ubicación de la información necesaria.

La creación de nodos y redes sobre temas de salud permitiría superar, en cierta medida, las limitaciones y dificultades que actualmente plantea el acceso a la información existente en este campo dentro del Internet. Permitiría, asimismo, desarrollar la EPS a un costo relativamente bajo, si se consideran el alcance territorial, la cantidad de información y el enorme número de usuarios que podrían aprovecharla.

El Internet ofrece una gran variedad de funciones, desde la simple comunicación por correo electrónico hasta teleconferencias, transmisión de imágenes y comunicación telefónica. Estos avances tecnológicos permiten buscar nuevas soluciones para viejos problemas en el área de la salud a un costo menor del que permitían las tecnologías tradicionales.

La consolidación de un nodo para temas de salud requiere la integración de múltiples instituciones y usuarios en torno al objetivo principal: de- 
sarrollar un sistema de EPS a distancia, a través del Internet, para el personal de la salud. Este objetivo se lograría por numerosos medios, desde la centralización de datos, hasta el proporcionamiento de acceso a procesos educativos interactivos, como teleconferencias, listas de discusión sobre temas de salud y otros núcleos de información, bibliotecas y bases de datos sobre temas médicos y sanitarios; la facilitación de la comunicación y el debate interinstitucionales en torno al establecimiento de normas (asistenciales, gerenciales, legales, etc.); la definición de procedimientos; la discusión y difusión de innovaciones científicas y técnicas; el apoyo a los sistemas de información, vigilancia epidemiológica y gestión institucional en los niveles nacional, provincial y local; y la facilitación de consultas entre expertos sobre casos clínicos.

\section{Antecedentes de la telecomunicación en el sector de la salud}

Durante los años sesenta y setenta se hizo patente la necesidad de transmitir a distancia información médica y sanitaria por medio de las redes de telecomunicaciones. Lo que por un lado constituía un adelanto tecnológico extraordinario apuntaba, simultáneamente, al deterioro de la relación entre médico y paciente y a la pérdida de subjetividad humana para dar paso a la fría objetividad de la tecnología. Este problema, aún no resuelto, debe ser motivo de reflexión siempre que se coloque en el Internet información de tipo sanitario.

La aparición en el campo de la medicina de las primeras tecnologías -el estetoscopio, el microscopio, los métodos de laboratorio para fines diagnósticos, etc.- señaló el comienzo de la separación entre médico y paciente. En 1878 apareció en Estados Unidos de América el primer anuncio de un médico que atendía consultas por teléfono y en 1880 se extendió el uso de este aparato para brindar atención durante las 24 horas del día. En 1905, William Einthoven exploró la posibilidad de transmitir a una gran distancia por vía telefónica la imagen de un electrocardiograma, proceso al que se denominó telecardiograma. En 1967 al fin se logró transmitir desde Francia un telecardiograma que fue interpretado en Washington, DC, y devuelto al lugar de origen en 15 segundos.

En 1968 se hizo un primer intento por usar la televisión para examinar a personas situadas a distancia y en esa misma década, a raíz de la Conferencia sobre Procesamiento de Datos Diagnósticos celebrada en 1959 en el Instituto Rockefeller de Nueva York, se produjeron las primeras tentativas por aplicar las computadoras al campo de la salud. Entre los aspectos más halagadores de la nueva informática figuraban la posibilidad de dar a los médicos acceso a centros de formación lejanos, la gran capacidad de almacenamiento de datos y la velocidad con que estos se podían encontrar. Sus ventajas se hicieron aun más patentes frente al crecimiento de los archivos hospitalarios, a las dificultades del almacenamiento de las fichas clínicas y al incesante flujo de datos estadísticos y epidemiológicos. Más tarde surgirían nuevas posibilidades, como la creación de sistemas computadorizados para diagnosticar enfermedades difíciles o interpretar radiografías y muestras microscópicas. Al compás de todo este desarrollo se despertaba en los trabajadores de la salud el temor, no infundado, a ser desplazados paulatinamente por las computadoras.

\section{El Internet y la salud}

El desarrollo tecnológico en materia de comunicaciones ha avanzado tan vertiginosamente en época reciente que ahora es posible transmitir grandes volúmenes de información a puntos remotos en fracciones de minutos. Este fenómeno supone la globalización de la información, ya que cualquier usuario con acceso a estos medios de comunicación electrónicos puede obtener la información que esté disponible en cualquier país del mundo. Estas últimas tecnologías también permiten una comunicación interactiva entre usuarios situados en diferentes países o territorios, independientemente de la distancia que los separe.

De todos estos adelantos tecnológicos, el Internet es el más importante, por tratarse de una gigantesca red de comunicación que enlaza a usuarios en el mundo entero por medio de miles de redes grandes o pequeñas alrededor del mundo y que brinda acceso a información profusa y sumamente diversa. El crecimiento del Internet en los últimos años ha sido explosivo. Esta red, que crece mensualmente a un promedio de un millón de suscriptores situados en todas partes, sigue creciendo desmesuradamente, ya que en ella se inscribe un usuario nuevo cada 2 minutos.

El Internet se ha convertido en un instrumento indispensable para profesionales, investigadores y técnicos en los diversos campos del saber. El desarrollo reciente de redes informáticas, como la World Wide Web (WWW), han sumado nuevas funciones a las que ya ofrecía el Internet (correo electrónico, talk, TELNET, etc.). Todas en su conjunto permiten transmitir a grandes distancias desde el mensaje más sencillo, hasta fotografías, libros, videos, catálogos, teleconferencias y "teletrabajos".

No obstante este desarrollo revolucionario, la plétora de información y su falta de orden hacen 
que muchos perciban el Internet como una Torre de Babel. En el campo de la salud, su principal aplicación ha sido, hasta ahora, en el área de la medicina asistencial, tanto en el ámbito público como en el privado, y su aprovechamiento ha sido más individual que institucional. Aún no se ha sacado provecho a su enorme potencial en el terreno de la regulación y el control estatales, ni en el de la epidemiología o de la organización y administración de servicios, por dar algunos ejemplos.

De hecho, las profundas transformaciones que están sufriendo los sistemas de salud nacionales exigen la capacitación del personal de nivel intermedio que trabaja en los servicios a un costo relativamente bajo y en un plazo breve, sin sacrificar la calidad de la capacitación. Al mismo tiempo, los avances científico-técnicos, cada vez más acelerados, dificultan la actualización de los profesionales y técnicos en los países latinoamericanos, proceso imprescindible para garantizar a la población su acceso a servicios de atención de calidad.

Se sobreentiende, en función de lo anterior, que un nodo de salud en el Internet tendría numerosos objetivos principales: concentrar y promover la producción de información en medicina y sanidad; facilitar la comunicación y el debate interinstitucionales en torno al establecimiento de normas asistenciales, gerenciales, legales, y demás; definir procedimientos; discutir y difundir innovaciones científicas y técnicas; fomentar la educación permanente del personal de los servicios de salud; brindar acceso a bibliotecas y bancos de datos; y apoyar los sistemas nacionales, provinciales y locales en lo referente a información, vigilancia epidemiológica y gestión institucional.

\section{¿Qué es el Internet?}

El Internet, como se explicaba anteriormente, es una vasta red de redes más pequeñas para el intercambio de información. Se desarrolló inicialmente como proyecto militar del gobierno de Estados Unidos, con el fin de lograr un sistema de comunicación descentralizado que no fuera blanco fácil de ataques enemigos. Con el tiempo la red pasó a la esfera civil, donde se mantuvo descentralizada hasta convertirse en un sistema igualitario, lo que significa que todos los usuarios tienen igual relieve dentro de la red. El Internet llegó a ser una herramienta de suma utilidad para instituciones académicas y agencias gubernamentales, y hasta hace algunos años la mayor parte de sus sitios de conexión pertenecían a centros de esta índole. Tan variados son los usos que permite la red, que actualmente el número de empresas comerciales suscritas a ella supera al de empresas no comerciales.
El Internet se compone de redes corporativas grandes y formales, como AT\&T y Digital Equipment, y también de redes pequeñas. En agosto de 1993 ya tenía más de 14000 redes, y según las estadísticas de aquel momento, un promedio de 1000 redes se incorporaban mensualmente al Internet. En enero de 1995 la cifra total había aumentado a 26274 redes. Ya había 84 países con acceso directo al Internet y aproximadamente 48 millones de usuarios. El Internet es, por lo tanto, la red más abierta del mundo, con miles de computadoras que ponen información almacenada al alcance de cualquier usuario.

\section{¿Qué es una red computadorizada?}

Una red computadorizada es un conjunto de computadoras enlazadas por conexiones físicas (cables, enlaces de radio, fibras ópticas). Consta de una computadora central donde se encuentra la mayor parte de la información; las computadoras de todos los usuarios constituyen el resto de la red. Esto no significa que la información esté controlada por alguna entidad o que haga falta una autorización para poder enviarla. La comunicación es horizontal y se produce de forma fluida entre todos los usuarios de la red, sin la intervención del operador de la computadora central, que se limita a manejar toda la red $\mathrm{y}$ a asegurar el funcionamiento general del sistema.

\section{¿Qué información se encuentra en el Internet?}

La información que proporciona el Internet suele ser de libre acceso o de dominio público, pero en ciertas situaciones es necesario obtener una autorización para tener acceso transitorio, bien sea gratuito o pagado. En general la información disponible se encuentra en documentos de instituciones gubernamentales y no gubernamentales, trabajos colocados por sus autores a disposición de los usuarios del Internet, bases de datos, listas de discusión y otras fuentes. En el campo de la salud se ofrece acceso, por citar un ejemplo, a datos del Centro Nacional de Cáncer del Japón, de la OPS/OMS, del Fondo de las Naciones Unidas para la Infancia, de La Administración de Alimentos y Medicamentos de los Estados Unidos y de escuelas de medicina y universidades prestigiosas en el mundo entero.

\section{¿Cómo circula la información en el Internet y cuánto cuesta?}

Las comunicaciones entre países y continentes se hacen por satélite. Hasta fines de 1995, la información que llegaba a América Latina pasaba 
por el llamado backbone (espina dorsal) o núcleo de la Red de la Fundación Nacional de Ciencias (NSF, por National Science Foundation) de los Estados Unidos. Cada país pagaba el costo de la conexión desde el punto de acceso en su territorio hasta el punto de entrada en Estados Unidos, pero el trayecto desde este último hasta el núcleo de la NSF y hasta los enlaces en el resto del mundo eran financiados por la propia NSF. Debido, sin embargo, al enorme tráfico comercial que circula por su núcleo, la NSF ha dejado de financiar estos pagos y está cediendo el núcleo a compañías comerciales, como la compañía telefónica MCI, que a su vez han creado puntos de acceso a la red (NAP, por el inglés network access points) de Internet.

Este último paso ha ocasionado cambios en las tarifas de cobro por actividades sin fines comerciales, como la educación y la investigación. Para las redes latinoamericanas, el aumento ha sido de US\$ 1000 a 2000 mensuales y se aplica a la conexión desde el punto de bajada de la señal de satélite en Estados Unidos hasta los puntos de acceso a la red, que son manejados por compañías comerciales.

\section{¿Qué servicios ofrece el Internet?}

El Internet proporciona múltiples servicios por medio de una gran variedad de herramientas, algunas de las cuales se describen a continuación.

Correo electrónico (E-mail). Este es, indiscutiblemente, el servicio usado con mayor frecuencia. Permite el intercambio de correspondencia entre millones de personas en el mundo entero y el envío no solo de documentos formales, sino de cualquier otra comunicación escrita.

Conversaciones en línea (talk). Otro uso que ofrece la red es la comunicación en tiempo real. Esto significa que personas situadas en ciudades, países o continentes diferentes pueden ver lo que escribe la otra persona en el momento exacto en que lo está escribiendo. Es una vía ideal para resolver consultas urgentes, especialmente entre personas separadas por grandes distancias y cuando se necesita que el mensaje sea claro y preciso o que haya una interacción, como en la docencia.

Acceso remoto (FTP y TELNET). Estos servicios permiten al usuario conectarse con cualquier computadora alrededor del mundo y trabajar como si estuviera sentado ante la computadora a la que se conectó.
Búsqueda de usuarios en todo el mundo (WHOIS). Ciertas funciones del Internet permiten localizar a personas conectadas a la red. A pesar de que hay varios millones de usuarios, es posible obtener la dirección electrónica y postal de cualquiera de ellos por medio de estas funciones.

Servidores de listas de discusión (listservers). Los servidores de listas cuentan con una dirección de correo electrónico y pueden manejar numerosas listas de discusión, a las que los usuarios, aunque estén en diferentes ciudades o países, se suscriben para participar en debates sobre temas específicos. Existen miles de listas de este tipo y cada día se crean listas adicionales, puesto que el requisito para la apertura de una lista es que un mínimo de 10 personas estén interesadas en un mismo tema. Estas personas pueden participar en los debates activamente o limitarse a ser observadores. Un listserver maneja varias listas o conferencias que se transmiten por correo electrónico. La información se envía a una dirección electrónica en particular y el servidor se encarga de distribuirla automáticamente a todos los integrantes de la lista.

El Gopher. Muchas computadoras tienen archivos de información que pueden explorarse libremente. Para facilitar esta tarea se ha creado el Gopher, que es un sistema de búsqueda y recuperación de documentos a base de menúes que los identifican por orden jerárquico. El Gopher es una vía de acceso a información sobre temas de salud, como alimentos, medicamentos, abuso de sustancias, avances terapéuticos, y muchos más. El servidor instalado en el nodo central agrupa distintas opciones en un solo menú y estas apuntan a otros servidores que a su vez proveen información sobre temas de salud. Cuando el usuario desea obtener información que no está disponible localmente, el servidor central automáticamente consulta a estos otros servidores.

World Wide Web. Este servicio da acceso a información académica presentada en "hipertexto" (hypertext, o texto resaltado). Si el usuario desea obtener información adicional sobre un tema en particular, debe oprimir el ratón mientras apunta a la palabra o al gráfico resaltado. Los servidores de la WWW dan acceso a tres tipos de documentos en hipertexto: servidores; programas a base de gráficos; e información agrupada que sirve de vía de acceso (gateway) a proveedores de recursos adicionales. La WWW se usa para recuperar información y tener acceso a enormes volúmenes de documentos. Es fácil de usar gracias a su interfaz gráfica y su 
funcionamiento es similar al del Gopher, ya que por medio del servidor local se tiene acceso a cientos de servidores dispersos por el mundo entero.

\section{El Internet aplicado al campo de la salud}

La promoción de un nodo de salud en el Internet tiene por objetivo facilitar a usuarios institucionales e individuales la transmisión y el intercambio de experiencias y conocimientos. Este proceso ya se está produciendo en algunos países, pero de manera poco organizada, lo cual obstaculiza el acceso a la información circulante. El enorme caudal de información médica y sanitaria y su amplia dispersión dificultan su obtención. Por otra parte, ubicar las bases de datos y listas de discusión requiere mucho tiempo. El usuario típicamente se limita a usar los servicios que va conociendo al azar.

Los nodos de salud pondrían al alcance de los usuarios información y materiales para su actualización y educación permanente en salud de una manera ordenada y sistemática por medio del Internet y de los sistemas que facilitan su utilización. Tales nodos constituirían, por consiguiente, un estímulo al debate, a la investigación y a la docencia. Las herramientas de comunicación, tanto horizontal como vertical (es decir, entre usuarios y entre nodos), se usarían para dar a conocer los últimos avances, solicitar información destinada a crear nuevos servicios o agrandar los ya existentes, o promover el debate. Los participantes pondrían a circular sus dudas y necesidades, generando de ese modo un sistema de EPS a distancia. Los servidores de información y la WWW ofrecen la posibilidad de extender los nodos.

Los medios señalados constituyen poderosas herramientas para dinamizar las instituciones de salud y movilizar al personal de los servicios mediante su integración a los debates que se producen en el ámbito académico y en el campo de la investigación. Asimismo, los planteamientos y reflexiones de estos individuos se verán enriquecidos por la interacción con quienes pueden, en el transcurso de la práctica cotidiana, ofrecer una perspectiva diferente de los problemas.

El desarrollo de la EPS a distancia se verá facilitado por el carácter interactivo de este tipo de comunicación, que no solo permite consultas entre usuarios, sino a bibliotecas y bases de datos en el mundo entero. El usuario simplemente baja (download) la información de su interés al disco de su computadora. Las posibilidades educativas comprenden desde la capacitación de personal técnico y auxiliar, hasta la formación continuada de posgrado (incluidos los niveles de maestría y doctorado) a través de programas desarrollados por instituciones académicas, científicas y gubernamentales, o de organismos internacionales supervisados por el nodo central. Las propuestas de capacitación en las diferentes disciplinas se colocarían en el servidor de dicho nodo.

El servidor WWW, que permite consultar archivos en el mundo entero por autor, tema o campo de actividad, es de enorme utilidad tanto en el gremio académico como en el de los servicios de salud, particularmente en términos de la actualización científico-técnica del personal.

Cabe señalar que el Internet, por ser un instrumento meramente técnico, no produce transformaciones por sí solo. Más bien, estas dependerán del proyecto en que se inscriba su uso, de la capacidad innovadora de quienes lo ejecuten y del seguimiento de las propuestas y cambios que se vayan produciendo.

El Internet por sí solo fomenta la actualización de los profesionales y técnicos con su mero uso, cuyo costo es relativamente bajo. Una vez identificada la necesidad de determinada información, se puede solicitar a varias instituciones, aunque estén situadas en distintos lugares, información que ha sido elaborada por todas en conjunto, es decir, enriquecida por los aportes de cada institución. El producto así elaborado puede quedar permanentemente incorporado al contenido del nodo.

Es importante percatarse de las ventajas que ofrece el Internet en términos de costo, ya que el acceso a información y la posibilidad de capacitar a un gran número de usuarios, sin necesidad de que se desplacen geográficamente, redunda en beneficios económicos y de tipo organizacional. Aunque por el momento no está muy desarrollada la capacidad informática de las instituciones de salud, y particularmente de los servicios asistenciales, los costos de desarrollarla serían relativamente pocos a la luz de sus grandes ventajas. También cabe considerar el ahorro en impresión y distribución de materiales.

Los participantes y destinatarios de un nodo de salud serían todas las instituciones que se desenvuelven en este campo; los profesionales y técnicos de la salud de los niveles central y local; investigadores y personal docente; alumnos de grado y posgrado de las diversas disciplinas en el área de la salud y en sus campos afines; y miembros de asociaciones científicas y de la seguridad social.

El desarrollo del nodo de salud. El desarrollo del nodo de salud se llevaría a cabo mediante un proceso de desarrollo tecnológico y otro de desarrollo institucional. El primero abarcaría la creación física del nodo y su conexión al Internet; el segundo, la instalación de diferentes servidores y la conexión de los usuarios a los nodos. El desarrollo institucio- 
nal incluiría, por lo tanto, los siguientes aspectos: instalar y promover servidores como el Gopher y la WWW a modo de concentrar la información disponible en la red de Internet; desarrollar información correspondiente a las distintas áreas contenidas en el nodo; crear y promover metodologías de EPS y de actualización de personal a distancia, utilizando imágenes y sonido para reforzar el carácter interactivo; detectar nuevas necesidades de información con miras a extender la red; fomentar el uso de las distintas vías de comunicación (E-mail, talk, TEL$N E T$ ) entre los destinatarios y participantes para favorecer el intercambio de experiencias e información; y promover el uso de los servidores y WWW a manera de ampliar la utilidad de las herramientas anteriores.

Desde el punto de vista metodológico, el nodo funcionaría como motor inicial y movilizador de los recursos ya disponibles, coordinándolos y desplegando las posibilidades que ofrece la red, en virtud de su carácter descentralizado, para crear y circular información.

La creación de un nodo que aproveche las herramientas disponibles para hacer más accesible la información implica un nivel de coordinación más refinado que el del Internet, que permita concentrar y organizar la información sin alterar el carácter descentralizado de la red. Esto exige que el nodo opere como centro de información dinámico, centralizando la información y descentralizándola de nuevo. Las instituciones participantes y destinatarias que están a la cabeza de los adelantos en el campo de la salud señalarían la necesidad de iniciar o estimular búsquedas específicas y de dar ordenamiento sistemático a toda nueva información.

Un personal actualizado es capaz de resolver problemas en el lugar en que estos se presentan, sin necesidad de traslados costosos por falta de información o de acceso a centros de consulta prestigiosos. Aun cuando el traslado es inevitable debido a la falta de la tecnología adecuada o de capacitación en un área específica, este medio ofrece al médico local la posibilidad de mantener un diálogo con los servicios en que ingresan sus pacientes.

La utilidad del nodo dependerá de la creación de una "cultura" en torno a su uso, es decir, de la presencia de usuarios que participen de forma interactiva y autónoma en la generación y reproducción de información, y de la promoción del trabajo en equipo. Las posibilidades se ampliarán en la medida en que el material incluido en el nodo fomente en los trabajadores, profesionales y técnicos una actitud activa y analítica hacia la búsqueda de soluciones.

El desarrollo de esta nueva forma de producir, difundir y usar información implica que los niveles de decisión deberán promover la capacidad informática de las instituciones no solo mediante la adquisición de tecnologías, sino también de la capacitación y estimulación intelectual del personal de salud.

El Internet ofrece, por medio de sus listas de interés, acceso a temas que raras veces suscitan una interacción y permite que países o zonas de menor desarrollo que enfrentan grandes dificultades para lograr transformaciones institucionales examinen la experiencia acumulada en otros lugares. La red también ofrece participación en teleconferencias, en las cuales una entidad (a menudo un experto en el tema central de la conferencia) coordina la información y las discusiones. Para cada conferencia se formula una serie de preguntas y el coordinador abre la discusión planteando un problema en forma de interrogante. Cada participante puede intervenir en la discusión desde su computadora tan pronto lee el planteamiento y todos los integrantes de la teleconferencia pueden participar en ella simultáneamente. Los temas se resumen en forma de menú en el directorio de la conferencia.

\section{Las experiencias de Nicaragua y Bolivia}

En Nicaragua y Bolivia ya se ha instalado y desarrollado un nodo de Internet destinado al sector de la salud. Un primer paso, como ha de ser siempre, fue la negociación con la compañía telefónica local, que en la mayor parte de los países de América Latina es una empresa privada o en proceso de privatización. La creación de un nodo independiente (Nicaragua) o regulado por una empresa que entrega una licencia al nodo (Bolivia) a menudo es decisión autónoma o depende de las condiciones de funcionamiento autorizadas por la empresa telefónica nacional. En cualquier caso se torna indispensable disponer de personal idóneo para administrar el nodo o contratar a una empresa que brinde este servicio a distancia.

En ambos países se presentó el problema de la poca capacitación del personal de salud en el campo de la informática, carencia que obliga a llevar a cabo procesos de capacitación intensos y prolongados. No obstante, la disponibilidad en español de software que es fácil de aprender (Winsocket) permite a una persona con conocimientos básicos de Windows aprender el uso básico de las herramientas del Internet en cuestión de 8 horas.

Por último, es fundamental, para llevar adelante la creación de un nodo en el área de salud, estimular y fomentar una cultura institucional respaldada por actividades de mercadeo entre las distintas instituciones que conforman el sector sanitario. Tanto en Nicaragua como en Bolivia se creó un nodo con información de interés nacional y se dis- 
tribuyeron boletines para los usuarios con datos sobre el uso, el funcionamiento y las aplicaciones del Internet en el área de la salud. El uso de Internet se estimuló, además, mediante la apertura de cuentas especiales para miembros del personal de salud.

\section{CONCLUSIONES}

El sector de la salud de la mayor parte de los países está pasando por transformaciones estructurales sin poseer la capacidad de procesar los cambios necesarios. Diversos problemas — dificultades financieras, una mayor demanda de servicios, salarios bajos, disminución de los planteles de personal, cambios en la organización de los servicios (descentralización, creación de sistemas locales, autogestión, etc.) - ponen de manifiesto la necesidad de desarrollar procesos de capacitación que no requieran alargar los horarios ni movilizar al personal; que favorezcan la formación de equipos; que involucren a un gran número de trabajadores en cada institución; y que permitan una interacción entre los servicios y los niveles normativos, gerenciales, académicos e investigadores a fin de romper el aislamiento en que tan frecuentemente operan unos y otros.

Todas las herramientas del Internet son idóneas para la educación a distancia, puesto que permiten el envío de material bibliográfico, ejercicios, objetivos de lectura y evaluaciones. El talk permite "conversaciones" por computadora entre alumnos y personal docente. El Gopher y la WWW ofrecen nuevas posibilidades tecnológicas aplicables a la EPS, entre ellas la de desarrollar cursos interactivos con participación de catedráticos y alumnos separados por enormes distancias.

Entre los posibles usos del Internet para fines de la EPS a distancia destacan los siguientes: la acreditación de instituciones de salud asistenciales o académicas que ofrezcan desarrollar cursos de educación continuada para profesionales; el ofrecimiento de cursos en una institución o varias, con asignación de miembros del personal docente para el seguimiento del proceso formativo; y la facilitación del envío de materiales educativos y comunicaciones que normalmente se mandarían por vía postal.

Paulatinamente se podrán introducir algunos cambios para abreviar la distancia entre el proceso de capacitación y el de evaluación (tanto del alumno como de la actividad de capacitación propuesta). Muchas veces surgen dificultades porque el personal no ve reflejadas en estos procesos sus inquietudes y problemas. Por otra parte, muchas propuestas educativas no tienen en cuenta las dificultades de comprensión de las personas a quienes se dirigen. Los cambios introducidos estarían orientados a facilitar, mediante núcleos de participantes y discusiones en grupo sobre los materiales educativos, el planteamiento de dudas, el análisis de las propuestas de capacitación y de su adecuación a las necesidades cotidianas y el establecimiento de una comunicación con la entidad capacitadora. Esto puede consistir en el envío por el correo electrónico de la actividad generada en grupo, o en "conversaciones" electrónicas con los miembros del personal docente. Sería también factible desarrollar metodologías menos estructuradas para conseguir una interacción más libre entre catedráticos y alumnos. Huelga decir que una tecnología como el Internet puede y debe adaptarse a las necesidades y particularidades de una gran variedad de situaciones.

Por último se advierte que la incorporación del Internet al sector sanitario como herramienta de trabajo no debe convertirse en asunto de expertos, ya que ello deformaría la naturaleza horizontal del Internet. Más bien, el uso de la red debe introducirse en todos los procesos que tienen lugar dentro de las instituciones de salud. Con tal fin, todo miembro del personal del sector sanitario debe aprender a manejar esta tecnología, sin que por ello se convierta en un ingeniero de informática.

La creación de un nodo de salud es un proceso lento. El Internet no es una tecnología efímera y su incorporación como herramienta de trabajo, en áreas tales como la vigilancia epidemiológica, la capacitación a distancia, y la consulta a bibliotecas y centros de documentación, redundará indudablemente en el mejor funcionamiento del sector de la salud.

\section{SYNOPSIS}

\section{The Internet and its incorporation by the health sector}

The governmental and health sector reforms that are being carried out in the countries of the Region demand the rapid training of health personnel to face the challenges posed by the process of change. This report explores the many possibilities of the Internet to serve as a mode of communication and updating of health professionals and technicians and as a vehicle for the dissemination of information on subjects of interest to scientists and researchers. 


\section{BIBLIOGRAFÍA}

Aiton JF. The World-Wide Web: an interface between research and teaching in bioinformatics". Dis-Markers 1994;12:3-10.

Anderson RK, Haddik A, McCray JC, Wunz TP. Developing a health information infrastructure for Arizona. Bull Med Libr Assoc 1994;82:396-400.

Barnett O, Shortliffe E, Chueh $\mathrm{H}$, et al. Patient care applications on Internet. Proc Annu Symp Comput Appl Med Care 1994; 1060.

Bax M. Getting on the Internet [editorial]. Dev Med Child Neurol 1995;37:565-566.

Belmonte-Serrano MA. Internet en la medicina del 2000. Med Clin Barc 1995;104: 744-752.

Bergren MD. III. Electronic communication. J Sch Nurs 1995;11:7-9.

Boutell T, Garrels JI, Franza BR, Monardo PJ, Latter GI. Ref52 on Global Gel Navigator: an internet-accessible two-dimensional gel electrophoresis database. Electrophoresis 1994;15: 1487-1490.

Brandon AN, Hill DR. Selected list of books and journals for the small medical library. Bull Med Libr Assoc 1995;83:151-175.

Breimer LH, Breimer DD. A computerbased international "thesis-line?" Trends Biochem Sci 1995;20:175-176.

Burnett L, Barger D. Nurse executives online: connecting to on-line services. I. Bulletin boards and the Internet. Aspens Advis Nurse Exec 1995;10(7):7-8.

Burnett L, Barger D. Nurse executives online: connecting to health case-related services. II. Bulletin boards and the Internet. Aspens Advis Nurse Exec 1995;10(8):6-8.

Cavanaugh CA, Yirka YM, Thompson CA Sending material for publication in AJPH via Internet. Am J Health Syst Pharm 1995; 52:1396-1399

Corn M, Johnson FE. Connecting the health sciences community to the Internet: the NLM/NSF grant program. Bull Med Libr Assoc 1994;82:392-395.

D'Alessandro MP, Galvin JR, Ekronen WE, et al. The virtual hospital: creating and organizing a ubiquitous health sciences organization on the Internet. Proc Annu Symp Comput Appl Med Care 1994:1061.

D'Alessandro MP, Lacey DL, Galvin JR, Erkonen WE, Santer DM. The networked multimedia textbook: distributing radiology multimedia information across the Internet. JR Am J Roentgenol 1994;163: 1233-1237.

Dailey DJ, Eno KR, Brinkley JF. Performance evaluation of a distance learning program. Proc Annu Symp Comput Appl Med Care 1994:76-80.

Datz FL, Baune DA, Christian PE. Redirection of client/server relationship of $X$ Window system as a simple, low-cost, departmental picture archiving and communication system solution for nuclear medicine. J Digit Imaging 1994;7:107-112.

Delamothe T. BMJ on the Internet. Br Med J 1995;310:1343-1344.
Di Giorgio CJ, Richert CA, Klatt E, Necich MJ. E-mail, the Internet, and information access technology in pathology. Semin Diagn Pathol 1994;11:294-304.

Dolin R. Medical applications on the Internet. MD Comput 1995; 12:162-165.

DoVan M, Humphrey LM, Cox G, Ravin CE. Initial experience with asynchronous transfer mode for use in a medical imaging network. J Digit Imaging 1995;8:43-48.

Ellenberger B. Navigating physician resources on the Internet. Can Med Assoc J 1995:152:1303-1307.

Erhardt Domino K, Pletcher T, Wilson W, Atkins D, Panko WB. The Internet: will this highway serve the digital library? Bull Med Libr Assoc 1994;82:426-433.

Finegold L. The Internet and the Journal [carta]. New Engl J Med 1995;333:1079.

Flannery MR. Cataloging Internet resources. Bull Med Libr Assoc 1995;83:211-215.

Frey $\mathrm{AH}$. The Internet biologist. FASEB I 1995;9:472.

Frey $\mathrm{AH}$. The Internet biologist [noticia]. FASEB J 1995;9:994.

Frey $\mathrm{AH}$. The Internet biologist [noticia]. FASEB J 1994;8:1110.

Fuchs R. Sequence analysis by electronic mail: a tool for accessing Internet e-mail servers. Comput Appl Biosci 1994;10: 413-417.

Fuller SS. Internet connectivity for hospitals and hospital libraries: strategies. Bull Med Libr Assoc 1995;83:32-36.

Galvin JR. D'Alessandro MP, Kurihara Y, Erkonen WE, Knutson TA, Lacey DL. Distributing an electronic thoracic imaging teaching file using the Internet, Mosaic, and personal computers. AJR Am J Roentgenol 1995;164:475-478.

Gardner RA. Toxicology abstracts, an electronic journal on the Internet. Toxicology 1995:99:219-228.

Gilas T, Schein M. General surgery Internet forum [carta]. Dis Colon Rectum 1995; 38:780.

Gilas T, Schien M. New Internet LISTSERV mailing list, SURGINET [carta]. J Am Coll Surg 1995;181:277.

Giles IG. A compendium of reviews in biochemistry and molecular biology published in the second half of 1993. Int Biochem 1994;26:1163-1201.

Gnassi JA, Bormel JI, Blewett DR, Kim RJ, Barnett GO. A medical information resource server: one stop shopping on the Internet. Proc Anпu Symp Comput Appl Med Care 1994;1025.

Godard P, Godard M. The Internet and the Journal. [Carta]. N Engl J Med 1995;333. 1078-1079.

Goldwein JW, Benjamin I. Internet-based medical information: time to take charge [editorial]. Ann Intern Med 1995;123: 152-153.

Goldwein JW, Benjamin I. The Internet and the Journal [carta]. N Engl J Med 1995; 333:1078.
Grimes DA. Introducing evidence-based medicine into a department of obstetrics and gynecology. Obstet Gynecol 1995;86: 451-457.

Hancock L. Nursing resources on the Internet. Aspens Advis Nurse Exec 1994;10:7-8.

Harper R. Access to DNA and protein databases on the Internet. Curr Opin Biotechnol 1994;5:4-18.

Harper R. World Wide Web resources for the biologist. Trends Genet 1995;6:223-228.

Heumann K, George D, Mewes HW. A new concept of sequence data distribution on wide area networks. Comput Appl Biosci 1994;10:519-526.

Hildebrandt F. Genetic renal diseases in children. Curr Opin Pediatr 1995;7:182-191.

Hollander SM, Lanier D. Orientation to the Internet for primary care health professionals. Bull Med Libr Assoc 1995;83:96-98.

Huntley AC, Conrad SJ. Internet tools in the medical classroom. Med Educ 1994;28: 508-512.

Jack D. Popular medical information on Internet [carta]. Lancet 1995;346:250.

Kaltenborn KF, Himmelmann GW. Scientific information and communication on the Internet. Med Klin 1995;90:428-434.

Krol E, ed. Conéctate al mundo INTERNET: guía y catálogo. México, DF: O'Reilly \& Associates, Inc; 1994.

Lacroix EM, Backus JE, Lyon BJ. Service providers and users discover the Internet. Bull Med Libr Assoc 1994;82:412-418.

Lief PD. The Internet and the Journal [carta] N Engl J Med 1995;333:1077.

Lilienthal MG. Defense Simulation Internet: next generation information highway. J Med Syst 1995;19:213-217.

Lindberg DA, Humphreys BL. The highperformance computing and communications program, the national information infrastructure and health care. J Am Med Inform Assoc 1995;2:156-159.

Lindberg DA. HPCC and the National Information Infrastructure: an overview. Bull Med Libr Assoc 1995;83:29-31.

Lindberg DA, Humphreys BL. Computers in medicine. JAMA 1995;273:1667-1668.

Ma J, Miyazaki S, Sugawara H. A handy database for culture collections worldwide: CCINFO-PC. Comput Appl Biosci 1995; 11:209-212.

McCoy K. Peeking through the Internet: getting started. Insight 1995;20:27-28.

McEnery KW. The Internet, World-Wide Web, and Mosaic: an overview. AJR Am J Roentgenol 1995;164:469-473.

McFarland MA, Gallagher K, Krash P, Bouchard C. Developing a health sciences library: more involved than meets the eye. Bull Med Libr Assoc 1995;83:216-220.

McKinney WP, Wagner JM, Bunton G, Kirk LM. A guide to Mosaic and the World Wide Web for physicians. MD Comput 1995;12:109-114,141.

McKinney WP, Barnas GP, Golub RM. The medical applications of the internet: infor- 
mational resources for research, education, and patient care. J Gen Intern Med 1994;9:627-634.

Mezrich RS, DeMarco JK, Negin S, Keller I, et al. Radiology on the information superhighway. Radiology 1995;195:73-81.

Millman A, Lee N, Kealy K. ABC of medical computing: the Internet. $\mathrm{Br}$ Med J 1995; 311:440-443.

Nakai K, Tokimori T, Ogiwara A, Uchiyama I, Niiyama T. Gnome-an Internet-based sequence analysis tool. Comput Appl Biosci 1994:10:547-550.

Nicholl D, Williams A, Davies D. BMJ on the internet: neuroscience on the internet. $\mathrm{Br}$ Med J 1995;310:1675.

Odaka T, Agata H, Furutani H, Ogura H. A general purpose neural network simulator system for medical data processing. J Med Syst 1994;18:305-314.

Odaka T, Takahama T, Wagatsuma H, Shimada $\mathrm{K}$, Ogura $\mathrm{H}$. A visual data analysis system for the medical image processing. J Med Syst 1994;18:151-157.

Owens RG. Ethics of executed person on Internet [carta]. Lancet 1995;345(8950):653.

Owens RG. Ethics of executed person on Internet [carta]. Lancet 1995;345(8949):653.

Pignone M. The Internet and the Journal [carta]. N Engl J Med 1995;333:1079.

Powsner SM, Roderer NK. Navigating the Internet. Bull Med Libr Assoc 1994;82: 419-425.

Rauch S, Holt MC, Horner M, Rambo N. Community hospitals and the Internet: lessons from pilot connections. Bull Med Libr Assoc 1994;82:401-406.

Regennitter FJ, Volz JE. An introduction to the Internet. Am J Orthod Dentofacial Orthop 1995;107(2):214-217.

Regennitter FJ, Volz JE. An introduction to the Internet. Am J Orthod Dentofacial Orthop 1995;107(3):339-344.

Regennitter FJ, Johnson BE. Electronic mail on the Internet. Am I Orthod Dentofacial Orthop 1995;108(2):219-222.
Reiser, JS, ed. La medicina y el imperio de la tecnología, México, DF: Fondo de Cultura Económica; 1990.

Reynolds TM. Popular medical information on Internet [carta]. Lancet 1995;346:250.

Richardson ML. A World-Wide Web radiology teaching file server on the Internet. Am J Roentgenol 1995;164:479-483.

Riley RA, Shipman BL. Building and maintaining a library: traditional skills applied to emerging resources. Bull Med Libr Assoc 1995;83:221-227.

Rizzolo MA, DuBois K. Developing AJN Network: transforming information to meet the needs of the future. Proc Annu Symp Comput Appl Med Care 1994;27-31.

Roeggla G, Landesmann U, Roeggla M Ethics of executed person on Internet [carta]. Lancet 1995;345(8944):360.

Rogers G. Nurses and the Internet. J Emerg Nurs 1995;21:160-162.

Ruskin KJ. How to access Journal of Clinical Monitoring abstracts on the Internet [carta]. J Clin Monit 1994;10:371-373.

Sanchez Ferrer A, Nunez Delicado E, Bru R. Software for reviewing biomolecules in three dimensions on the Internet. Trends Biochem Sci 1995;20:286-288.

Scherrer JR. Communications, future needs and present solutions. Int J Biomed Comput 1995;

Sharma P. Popular medical information on Internet [carta]. Lancet 1995;346:250.

Sorensen E. Internet-nyttig hjelpemiddel for leger. Tidsskr Nor Laegeforen 1994;114: 3350.

Spitz R. A short tutorial: the Internet as a global information source. Int J Clin Monit Comput 1995;12:43.

Steuer J. How to access images on the Internet. J Nucl Med 1995;36:24N.

Taubes G. Indexing the Internet [noticia] Science 1995;269: 1354-1356.

Tomaiuolo NG. Accessing nursing resources on the Internet. Comput Nurs 1995;13: 159-164.
Tyler JM. The Internet: legal rights and responsibilities. Med Surg Nurs 1995;4: 229-233.

Vaitones V, Rice L, Degner LF, Morra ME, Greening K, Fernsler J. "Protocol shopping" on the Internet. Cancer Pract 1995;3: 274-278.

Varma DG, Grossman J, Draper EJ, Durke VW III. Computerized scientific exhibit in radiology: a valuable format for delivering scientific infomation. Radiographics 1994;14:1127-1138.

Wallis JW, Miller MM, Miller TR, Vreeland $\mathrm{TH}$. An Internet-based nuclear medicine teaching file. J Nucl Med 1995;36: 1520-1527.

Watson CB. The Internet and the Journal [carta]. N Engl J Med 1995;333(16):1077.

Webster C, Pople A, Silva R, Wang X, McLinden S. A Tcl/Tk-based graphical interface to medical and administrative information. Proc Annu Symp Comput Appl Med Care 1994;992.

White M, Ostbye T. Global public health and the information superhighway. Epidemiologists are using the Internet [carta]. $\mathrm{Br}$ Med J 1994;309:736.

Wiviott LD. The Internet and the Journal [carta]. N Engl J Med 1995;333(16):1078.

Wood EH. The options for health professionals. J Am Med Inform Assoc 1994;1: 372-380.

Yoshihara H. Status quo and future prospects of the total hospital information system of a Japanese medical college. J Med Syst 1994;18:229-240.

Zelingher J. Exploring the Internet. $M D$ Comput 1995;12:100-108, 144

Zernik JH. Electronic Study Club on Internet [carta]. Am J Orthod Dentofacial Orthop 1994;106:21A-22A. 